\title{
Wireless Sensor Network for Monitoring \& Control of Environmental Factors using Arduino
}

\author{
https://doi.org/10.3991/ijim.v12i2.7415 \\ Jay P. Sipani $\left.{ }^{\bowtie}\right)$, Riki H. Patel $\left.{ }^{\bowtie}\right)$, Trushit Upadhyaya, Arpan Desai \\ Charotar University of Science and Technology, Changa, India \\ gopro.jaysipani16@gmail.com \\ rikipatel.ec@charusat.ac.in
}

\begin{abstract}
Today there is demand to monitor environmental factors almost in all research institutes, industries and even for domestic purpose. The analog data measurement requires manual effort to note readings and there may be a possibility of human error. Such type of systems fails to provide and store precise values of parameters with high accuracy. Analog systems are also having a drawback of storage/memory. Therefore, there is an increasing demand of a smart system which is fully automated, accurate and capable enough to monitor all the environmental parameters with utmost possible accuracy. In this paper a Wireless Temperature and Humidity Monitoring and Control system is presented which continuously scans the temperature and humidity conditions of surroundings and controls the same by triggering a cooling system. This system is interfaced with the GSM module which captures the undesired change in temperature through sensor and sends a warning message to the user acknowledging about the accurate and real-time remote temperature change and at the same time it triggers the cooling system to make the temperature condition to desirable one. This system is convenient to use with a good interface and operates intuitively for shorter ranges.
\end{abstract}

Keywords-Wireless; Arduino; temperature; humidity; GSM

\section{Introduction}

Field of monitoring and remote sensing has been revolutionized by wireless sensor network [1]. Wireless sensor networks can collect data from different sensors such as temperature, humidity, voltage, current etc. from remote locations and co-operatively pass the data through the network to the control station (or where data analysis is needed). Hence, wireless sensor networks can be used for monitoring of power data even from remote locations [2-3]. Continuous monitoring of these physical quantities online from remote control station helps to co-ordinate the uninterrupted operation in the process plants, industries and even in domestic utilities [4-5]. Keeping these situations in view, an attempt has been made in this project to monitor data through wireless sensor network for measurement of temperature and humidity [6-7]. All the measured data is transmitted from the site to the mobile device (control station) 
through SMS. The experimental setup includes temperature and humidity sensor (DHT11), LCD, SIM900A GSM Module. The codes developed are simulated and tested using Arduino IDE software and Arduino Uno board [8-9]. This way a wireless system is designed which is autonomous and can monitor as well as control the physical or environmental conditions, such as temperature and humidity and could stream the respective data to the control station [10-14]. The wireless sensor network proposed here is bidirectional which enables the control over the sensor activity. This system consists of various units which are assembled as a whole and works in rhythm for accurate analysis functioning.

The proposed system gathers the temperature and humidity parameters in various premises through wireless sensor networks which ranges from $0^{\circ} \mathrm{C}$ to $50^{\circ} \mathrm{C}$ and $20 \%$ $90 \% \mathrm{RH}$ respectively. As this system is equipped with sensors having high sensitivity along with low delay and accuracy governed components; this system is highly reliable for various applications like temperature monitoring and computer peripheral thermal protection, weather station applications as well as greenhouse monitoring system. [14-19].

\section{$2 \quad$ Methodology}

The successful implementation of proposed wireless sensor network includes temperature and humidity sensor (DHT11) in the premises where the online monitoring is done. The sensor is interfaced with the Arduino UNO development board. The programming in the Arduino board converts the analog output of sensor into digital form. The digital values of temperature and humidity are displayed on LCD. After that, the digital information is given to the GSM Module which is interfaced as a serial communication peripheral with Arduino. The GSM Module interfaced with Arduino will transmit the data to the mobile device through SMS. Additionally, a threshold temperature is set in the Arduino through programming which analyze the rise in temperature beyond threshold. As soon as the threshold value is reached; the cooling peripherals (5V DC Motor acting as a Fan) are automatically triggered to maintain the temperature and a warning SMS is send to the Mobile device along with displaying the value of same on the LCD. The Arduino and GSM shield are powered by DC Adapters/USB chords. Rest all the components in setup are powered by the Arduino ( $+5 \mathrm{~V})$.

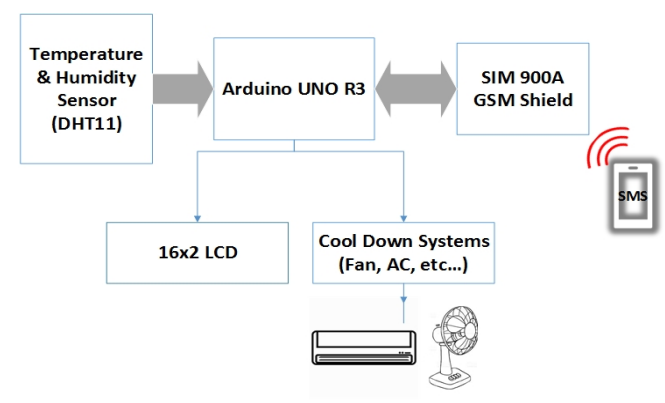

Fig. 1. Block Diagram of System [2] 


\section{Experimental Setup}

Block diagram of the system is as shown in figure 1. The experimental setup consisting of:

- Sensing unit - Temperature \& Humidity Sensor (DHT11)

- Processing unit - A Microcontroller (ATMEGA 328P)

- Communication unit - GSM shield (Transmitter) and Mobile device (Receiver)

- Power unit - Battery

is developed to measure and monitor temperature and humidity parameters in various environments. The brief about the hardware modules interfaced in the setup is as below:

\subsection{Temperature \& Humidity Sensor (DHT11)}

Fig. 2 shows DHT11 (Digital Humidity and Temperature) sensor which senses the temperature and humidity of the surrounding and gives a calibrated digital signal output. The sensor includes a resistive type humidity measurement component along with an NTC temperature measurement component. It is connected to an 8-bit microcontroller for high performance which offers excellent quality, fast response and antiinterference ability. It has a low power operating range $(3 \mathrm{~V}-5 \mathrm{~V})$. The component is $3-$ pin single row package and the major feature of this sensor is that the data for both temperature and humidity is available on a single data pin of the sensor.

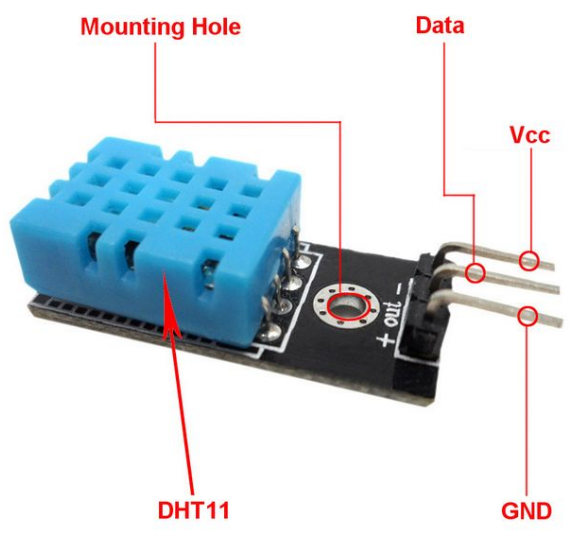

Fig. 2. DHT11 Sensor [1]

Table 1. Technical Specs Overview of DHT11

\begin{tabular}{|c|c|c|c|c|}
\hline $\begin{array}{c}\text { Measurement } \\
\text { Range }\end{array}$ & $\begin{array}{c}\text { Humidity } \\
\text { Accuracy }\end{array}$ & $\begin{array}{c}\text { Temp. } \\
\text { Accuracy }\end{array}$ & Resolution & Package \\
\hline $20-90 \% \mathrm{RH} 0-50{ }^{\circ} \mathrm{C}$ & $\pm 5 \% \mathrm{RH}$ & $\pm 2{ }^{\circ} \mathrm{C}$ & 1 & 4 Pin Single Row \\
\hline
\end{tabular}




\subsection{Arduino UNO Development Board}

Fig. 3 shows an Arduino which is an open-source prototyping platform. Arduino boards are able to receive inputs and can convert into relevant output. It contains an on-board power supply, USB port to communicate with PC, and an ATMEL microcontroller chip. It simplifies the process of creating any control system by providing the standard board that can be programmed and connected to the system without the need of any sophisticated PCB design and implementation. It is inexpensive, opensource and extensible, cross-platform and has a clear IDE.

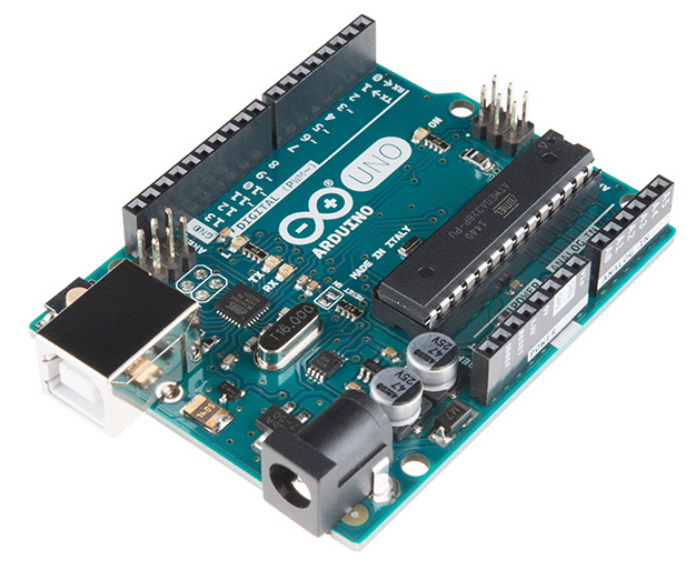

Fig. 3. Arduino UNO R3 [2]

Fig. 4 shows ATMEGA328P which is an Atmel 8-bit AVR RISC-based microcontroller.

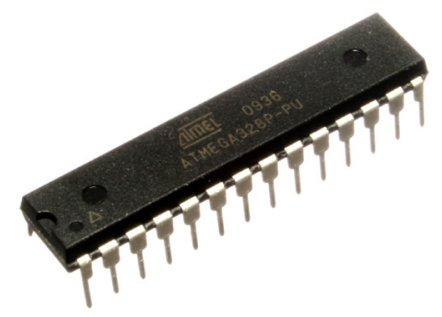

Fig. 4. ATMEGA 328P AVR Microcontroller [3]

Reasons to choose Arduino UNO R3:

- Simple to use and has on board debugger.

- Much cheaper than other development boards available in market.

- Consumes very less power and delivers greater accurate results.

- It supports variety of sensor modules readily available to be interfaced with it. 


\subsection{SIM900A GSM Shield}

Fig. 5 shows SIM900 which is designed with a very powerful single-chip processor integrating AMR926EJ-S core. It is a Quad - band (850/ 900/ 1800/ $1900 \mathrm{MHz})$ GSM/GPRS module with a size of $24 \mathrm{mmx} 24 \mathrm{mmx} 3 \mathrm{~mm}$ with SMT type suit for customer application. It has an embedded Powerful TCP/IP protocol stack. It can communicate with microcontrollers via AT commands. This module supports software power on and reset.

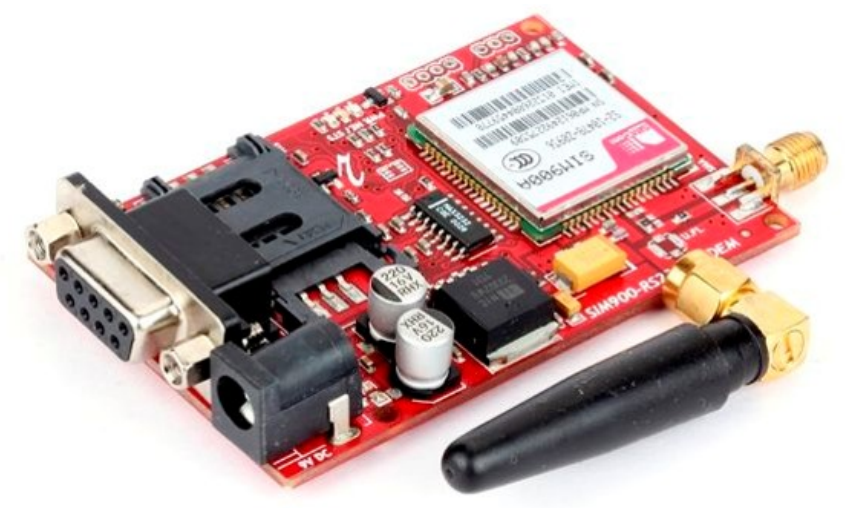

Fig. 5. SIM900A GSM Module [2]

Table 2. General Specs of SIM900A GSM Module

\begin{tabular}{|l|l|}
\hline PCB size & $71.4 \mathrm{~mm}$ X 66.0mm X 1.6mm \\
\hline Indicators & PWR, status LED, net LED \\
\hline Power Supply & $12 \mathrm{~V}$ \\
\hline $\begin{array}{l}\text { Communication } \\
\text { Protocol }\end{array}$ & UART \\
\hline RoHS & Yes \\
\hline
\end{tabular}

Reasons to choose SIM900A GSM Shield

- Supports GPRS.

- Better specifications than ZigBee, which is also a Wireless Communication Module.

- Easy to operate using AT commands and can be interfaced easily with any development board.

\subsection{6x2 Liquid Crystal Display (LCD)}

Fig. 6 shows an LCD (Liquid Crystal Display) which is used in all the electronics projects to display the status of the process. A $16 \times 2$ alphanumeric LCD is most widely used module of LCD nowadays. There are several other types of LCD available in the market also. The LCD are low cost, easily programmable, displays considerable 
number of characters and compatible with almost all microprocessor and microcontroller. 16x2 LCD has two horizontal line comprising a space of 16 displaying character. It has two inbuilt registers:

a) Command Register

b) Data Register

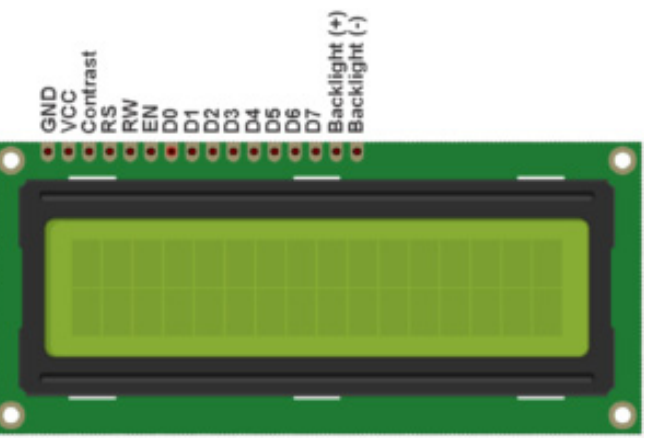

Fig. 6. $16 \times 2$ LCD [5]

\section{$4 \quad$ System Design}

The hardware setup of the proposed system is as shown in Fig. 7.

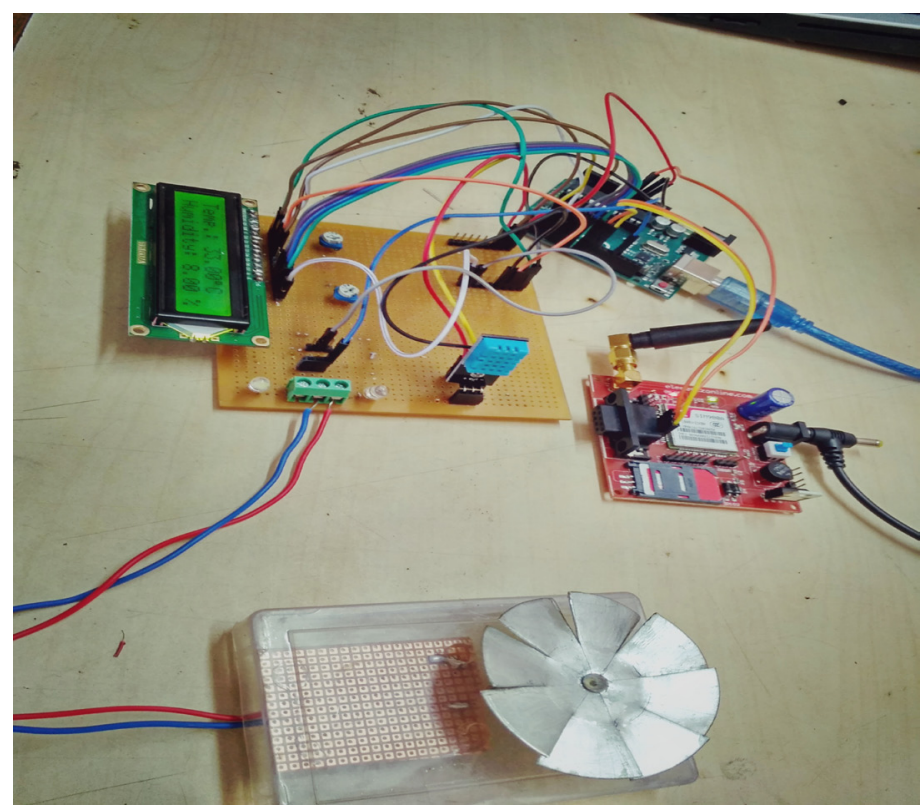

Fig. 7. Hardware Setup of System 
The DHT11 sensor scans the temperature and humidity of the surroundings and displays it on the LCD in real time with the help of Arduino. An SMS is sent every 30 minutes which contains the temperature and humidity information during that time. If the temperature rises beyond the threshold level, a warning message is displayed on LCD and an alert SMS is sent to the mobile number as well as the Arduino triggers the cooling systems to bring back the temperature to normal.

\section{$5 \quad$ Results and Discussions}

The temperature and humidity of various premises have been measured and the analysis of the data acquired from the project is carried out over here. Various scenarios are presented over here for the setup in an environment and data has been monitored for the same. The threshold temperature is set to $35^{\circ} \mathrm{C}$ which means that if the DHT11 sensor detects a temperature greater than $35{ }^{\circ} \mathrm{C}$, then the microcontroller sends the response signal to the cooling devices connected to it and initializes the process to maintain the temperature below the threshold.

\subsection{Case 1: When temperature is below the threshold temperature $\left(35^{\circ} \mathrm{C}\right)$}

When the temperature of the nearby premises temperature is below the threshold, it sends a text message to the mobile device indicating the present temperature and humidity statistics (SMS is sent every 30 minutes acknowledging about the temperature and humidity during that time). This way the system is under fully steady condition as there is no need to activate the cooling systems.

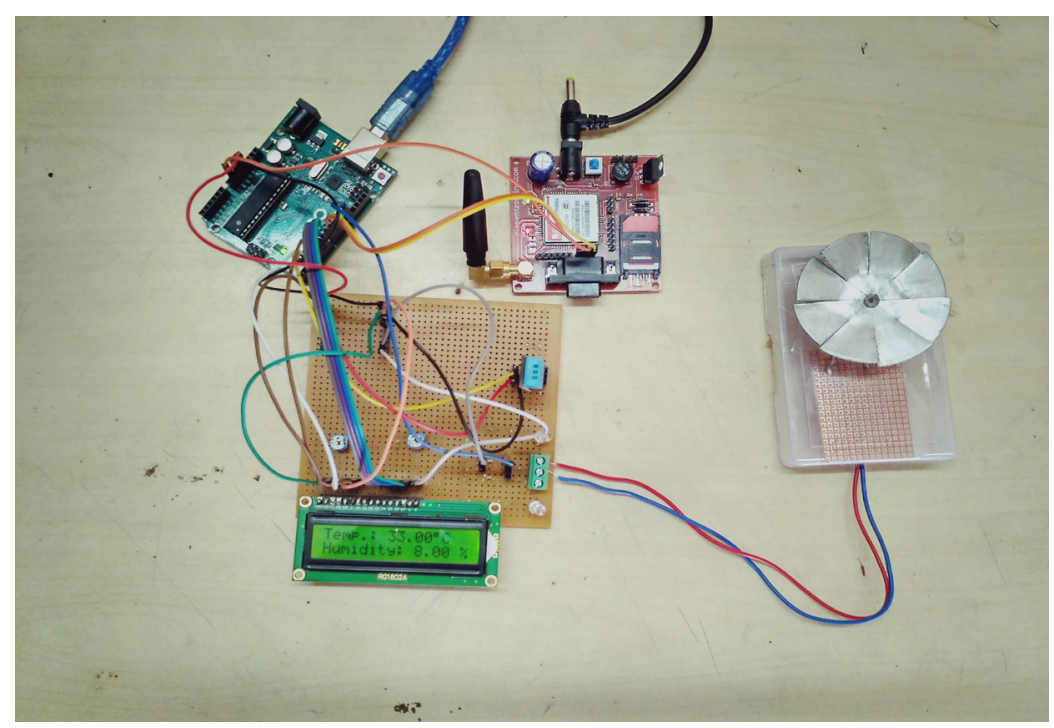

Fig. 8. System in Steady State 
The Fig. 8 shows that the system is in steady state condition which means the DHT11 sensor is sensing the temperature below the threshold limit and therefore the cooling system is in OFF state and an acknowledgment message about the temperature and humidity stats are sent to the mobile device during the boot-up of the system as shown in Fig. 9.

The message received on the mobile device is as shown in Fig. 10 which gives the reading of temperature and humidity of the surrounding environment.

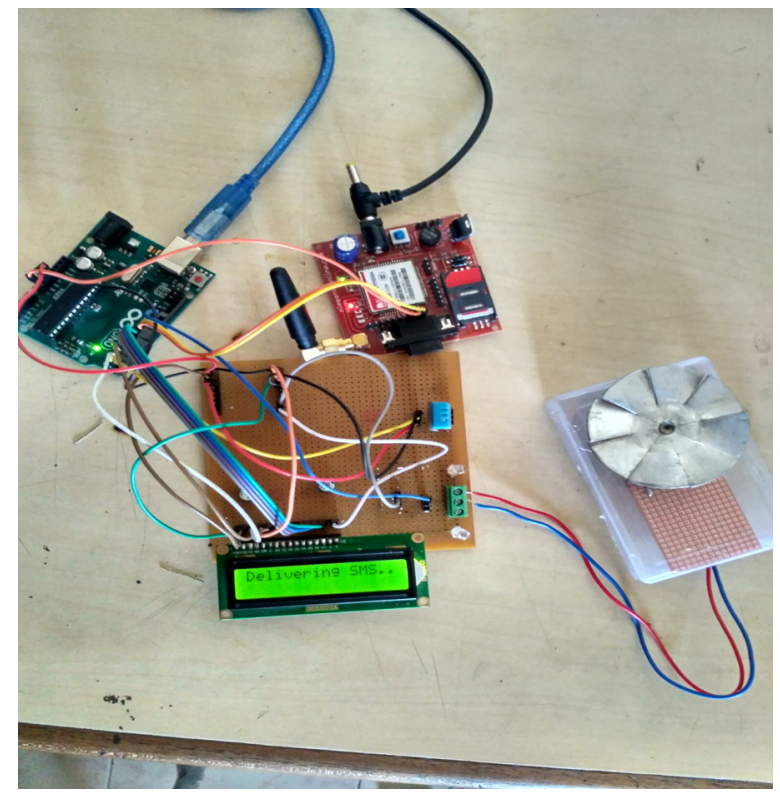

Fig. 9. SMS delivering process of system

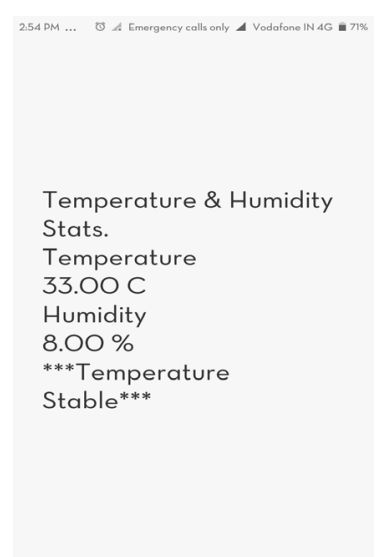

Fig. 10.Screenshot of SMS received on Mobile 


\subsection{Case 2: When temperature is beyond the threshold temperature $\left(35^{\circ} \mathrm{C}\right)$}

As the temperature of the surrounding increases (by means of putting a candle flame away at a distance of about $5 \mathrm{~cm}$ from the sensor), an increase in the temperature is marked with respect to time. The moment temperature crosses the threshold temperature of $35^{\circ} \mathrm{C}$, the Arduino sends a response signal to the cooling systems as shown in Fig. 11 which is initialized to cool the surroundings and at the same time SMS is sent to mobile giving warning message of temperature rise as shown in Fig. 12.

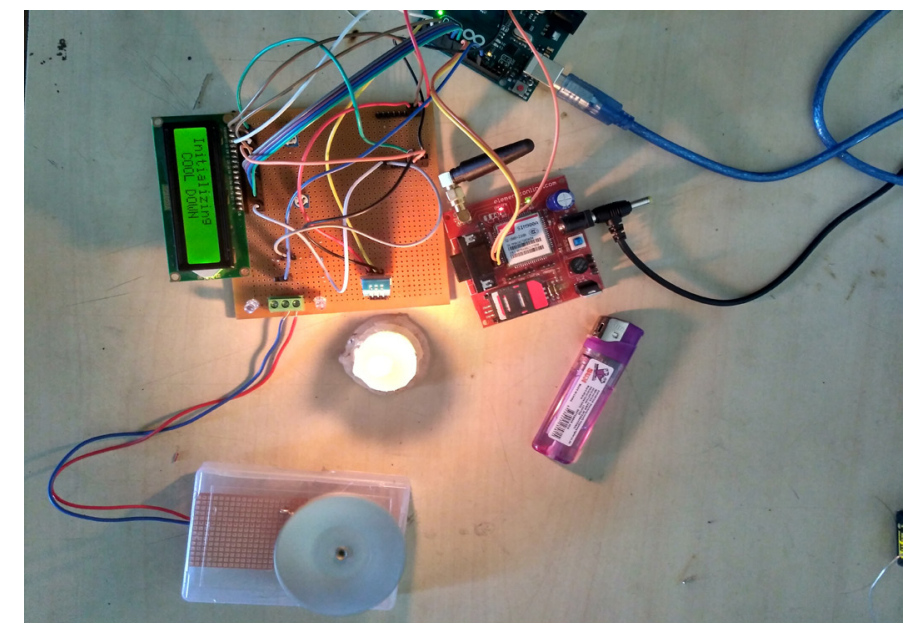

Fig. 11.Cool down process Initialized when temperature is raised

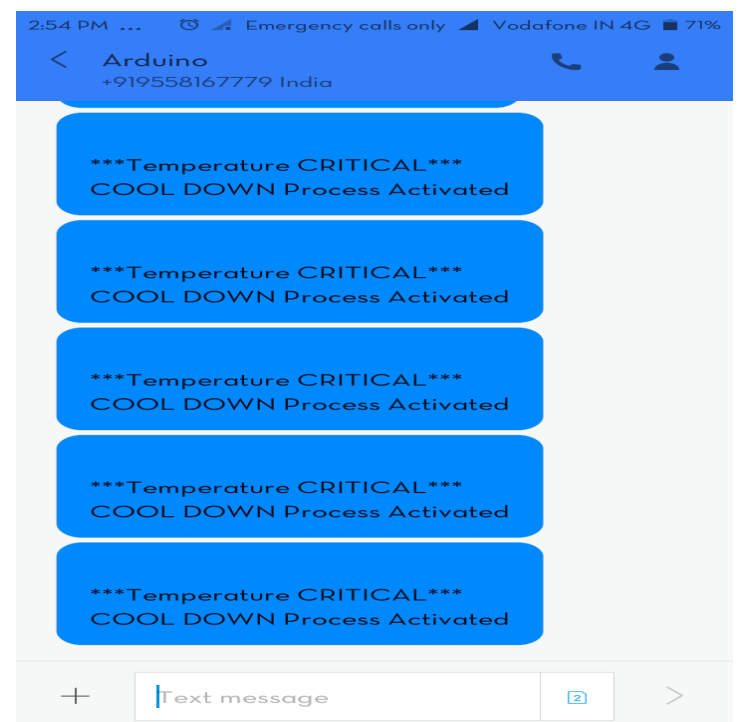

Fig. 12.Screenshot of SMS received during critical rise in temperature 
The chain of messages is stopped when the temperature is brought back to normal by cooling devices and the system is put back again to steady state condition. Once the steady state condition is reached; the cooling systems are deactivated as shown in Fig. 13.

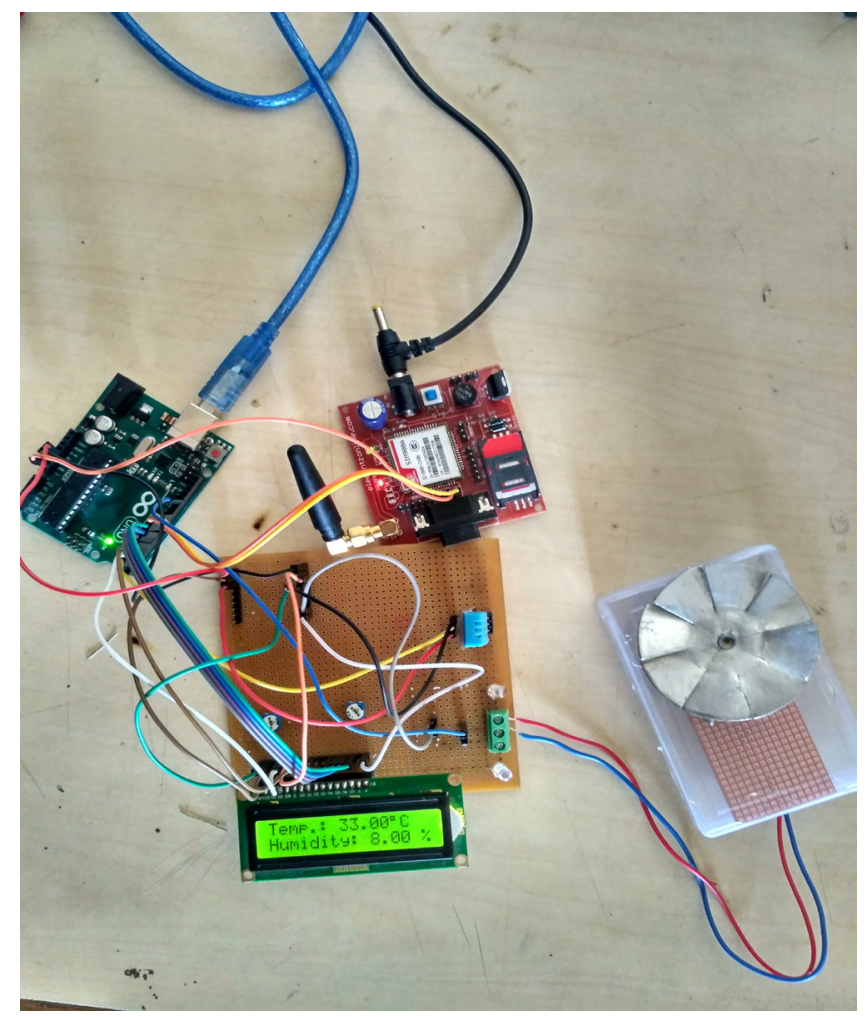

Fig. 13. System returned in Steady state

The above work proposes that the sensor works with a sensitivity of $\pm 2^{\circ} \mathrm{C}$ for temperature and $\pm 5 \% \mathrm{RH}$ for humidity and is capable of measuring the temperature and humidity values between $0^{\circ} \mathrm{C}$ to $50^{\circ} \mathrm{C}$ and $20 \%$ to $90 \% \mathrm{RH}$ respectively which makes the system applicable for HVAC (Heating, Ventilation and Air Conditioning) Systems as well as Home Automation Systems. The system proposed is competent and reliable for real time monitoring of temperature and humidity. As this system is totally digital, there is full arrangement of storage of the data, so it can be further used for analysis purpose. Also as the system is continuously monitoring the environment parameters, the user is updated at every fixed interval of time about the change in the parameters. Thus, user is aware about the changes in the parameters all the time. The disadvantage of this system is that it is developed only for small area. Network problem can lead to failure of message sending and receiving. Though the sensor is calibrated, there are chances of error in the readings as the sensor may get damaged when exposed to higher temperature beyond its limit. 


\section{Future Scope}

In future, this system would be upgraded to web-based monitoring system by using the GPRS technique which would ease the user to have access over the system remotely over the internet. Also, an upgrade for the monitoring of larger area would be done. Additionally, sensors like barometric pressure sensor, gas detector for air quality check with web interface can be integrated into a single system.

\section{Conclusion}

The proposed wireless sensor network provides a convenient method for effective monitoring of temperature and humidity in real time. This system is compact to an extent and cost effective as compared to other systems used for measuring the environmental factors. The system proposed here ensures that the nested wired systems can be replaced by the wireless sensor networks to get an accurate data as well as to avoid many hazardous issues.

\section{$8 \quad$ References}

[1] Hongguang Xu,Faming Li,Chunyu Chen,Xianhong Dong. NOKIA mobile phone LCD module LPH7366 principle and its application. Foreign electronic components. pp 5557,2004

[2] Dong, Wei, et al. "Mosaic: Towards city scale sensing with mobile sensor networks." Parallel and Distributed Systems (ICPADS), 2015 IEEE 21st International Conference on. IEEE, 2015.

[3] Patel, Riki H., Arpan Desai, and Trushit Upadhyaya. "A discussion on electrically small antenna property." Microwave and Optical Technology Letters 57.10 (2015): 2386-2388. https://doi.org/10.1002/mop.29335

[4] David, Chavez M., et al. "A low-cost, rapid-deployment and energy-autonomous wireless sensor network for air quality monitoring." Sensing Technology (ICST), 2015 9th International Conference on. IEEE, 2015. https://doi.org/10.1109/ICSensT.2015.7438376

[5] Upadhyaya TK, Kosta S, Jyoti R, Palandoken M; Negative refractive index materialinspired 90-deg electrically tilted ultra-wideband resonator. Opt. Eng. 0001;53(10):107104. https://doi.org/10.1117/1.OE.53.10.107104

[6] Shanko, Eriola J., and Michalis G. Papoutsidakis. "Real time health monitoring and wireless transmission: A $\mu$ Controller application to improve human medical needs." E-Health and Bioengineering Conference (EHB), 2013. IEEE, 2013.

[7] Upadhyaya, T.K., Kosta, S.P., Jyoti, R. and Palandöken, M., 2016. Novel stacked $\mu$ negative material-loaded antenna for satellite applications. International Journal of Microwave and Wireless Technologies, 8(2), pp.229-235. https://doi.org/10.1017/S17590787140 $\underline{0138 X}$

[8] Ellis, Trevor M., et al. "Investigation of outbreaks of highly pathogenic H5N1 avian influenza in waterfowl and wild birds in Hong Kong in late 2002." Avian Pathology 33.5 (2004): 492-505. https://doi.org/10.1080/03079450400003601

[9] Patel, Riki H., et al. "Design of S-Shape GPS Application Electrically Small Antenna." World Academy of Science, Engineering and Technology, International Journal of Electri- 
cal, Computer, Energetic, Electronic and Communication Engineering 9.4 (2015): 480483.

[10] UNO, ARDUINO. "DEVELOPMENT OF ROBOTIC ARM USING."

[11] Bacci, C., et al. "Preliminary Result of Frascati (ADONE) on the Nature of a New 3.1GeV Particle Produced in e+ e- Annihilation." Physical Review Letters 33.23 (1974): 1408.

[12] Dalsania, Piyush, et al. "Analysis of multiband behaviour on square patch fractal antenna." Communication Systems and Network Technologies (CSNT), 2012 International Conference on. IEEE, 2012. https://doi.org/10.1109/CSNT.2012.26

[13] Salford, Leif G., et al. "Nerve cell damage in mammalian brain after exposure to microwaves from GSM mobile phones." Environmental health perspectives111.7 (2003): 881.

[14] Upadhyaya, Trushit K., et al. "Miniaturization of tri band patch antenna using metamaterials." Computational Intelligence and Communication Networks (CICN), 2012 Fourth International Conference on. IEEE, 2012.

[15] Kosta Shiv Prasad, et al. "Human blood-based electronic transistor." International Journal of medical engineering and informatics 4.4 (2012): 373-386. https://doi.org/10.1504/ IJMEI.2012.050278

[16] Riki H Patel, Arpan H Desai, Trushit Upadhyaya, "Design Of H-Shape X-Band Application Electrically Small Antenna", International Journal of Electrical Electronics and Data Communication (IJEEDC), Volume-3 , Issue-12. Pp 1-4, 2015 IRAJ DOI Number IJEEDC-TRAJ- DOI-3486

[17] Markovà, Eva, et al. "Microwaves from GSM mobile telephones affect 53BP1 and $\gamma$ H2AX foci in human lymphocytes from hypersensitive and healthy persons." Environmental health perspectives 113.9 (2005): 1172.

[18] Patel Riki H., Hardik Modi, and Vrunda S. Patel. "Mobile Effect on Human Body." (2015).

[19] Mortazavi, S. M. J., et al. "Increased radio resistance to lethal doses of gamma rays in mice and rats after exposure to microwave radiation emitted by a GSM mobile phone simulator." Dose-response 11.2 (2013): dose-response.

\section{Authors}

Jay P. Sipani, Riki H. Patel, Trushit Upadhyaya, and Arpan Desai are with Charotar University of Science and Technology, Changa, India.

Article submitted 11 July 2017. Resubmitted 12 December 2017. Final acceptance 05 March 2018. Final version published as submitted by the authors. 